\title{
Maquiagem como estratégia de ensino e pesquisa em Odontologia Legal: relato de experiência
}

\author{
Mário Marques Fernandes*; Juan Antonio Cobo Plana**; Fernanda Capurucho Horta Bouchardet***; \\ Nelson Massanobu Sakaguti*; Gabriela Cauduro Rosa*; Rosane Pérez Baldasso*; Rogério Nogueira \\ Oliveira*
}

* Departamento de Odontologia Social, Faculdade de Odontologia da Universidade de São Paulo, São Paulo

** Instituto Médico Legal de Aragón, Zaragoza, Espanha

*** Departamento de Odontologia, Pontifícia Universidade Católica de Minas Gerais, Belo Horizonte

Recebido em 20/03/2017. Aprovado em 08/05/2017.

\begin{abstract}
RESUMO
O ensino da valoração de danos odontológicos pós-traumáticos é ministrado, em muitas situações, de forma teórica e ilustrativa com imagens projetadas, distanciando o aluno da vivência prática desse conteúdo. Por esse motivo, a simulação de lesões por meio de maquiagem pode auxiliar o ensino desse tema e também pesquisas, considerando, por exemplo, a necessidade de comparar a vítima de trauma com o seu estado anterior. $\mathrm{O}$ objetivo deste artigo é relatar uma experiência docente junto à graduação em Odontologia na Faculdade de Odontologia da Universidade de São Paulo, evidenciando o potencial uso da simulação realística com maquiagem no ensino da valoração de danos póstraumáticos e incitando a possível utilização em pesquisas na área de Odontologia Legal. Trata-se de uma metodologia inovadora, com perspectiva promissora no campo da Odontologia Legal.

Descritores: Avaliação de Danos. Metodologias de Avaliação de Danos. Odontologia Legal.
\end{abstract} Estética. Responsabilidade Legal.

\section{INTRODUÇÃO}

A temática humanista, ética e legal está contemplada nas atuais Diretrizes Curriculares Nacionais para os Cursos de Graduação em Odontologia, buscando estabelecer um perfil do egresso "Capacitado ao exercício de atividades referentes à saúde bucal da população, pautado em princípios éticos, legais e na compreensão da realidade social, cultural e econômica do seu meio, dirigindo sua atuação para a transformação da realidade em benefício da sociedade". Essas capacidades podem ser desenvolvidas em qualquer espaço de ensino, porém os componentes das Ciências Humanas e Sociais, os quais possuem denominações e conteúdos variados nas diferentes Instituições de Ensino Superior (IES), como Saúde Coletiva, Deontologia, Bioética, Ciências Sociais em Saúde, Odontologia Legal, Estágios Vivenciais e Odontologia do Trabalho, propiciam momentos 
privilegiados para tal aprendizado, devido às suas características e conteúdos específicos ${ }^{1}$.

Os temas de Deontologia e Odontologia Legal muitas vezes se apresentam áridos aos estudantes de graduação, pois projetam as questões jurídicas e periciais longe da futura realidade profissional, sendo ministrados por meio de metodologias ativas na tentativa de torná-los tão realistas quanto possível ${ }^{2,3}$.

Dentre as estratégias educacionais evidencia-se a simulação realística por meio de maquiagem (SRM), a qual pode ser usada como um recurso eficaz quando um quadro clínico é pouco frequente na rotina ambulatorial, ou mesmo pericial. Como exemplos de SRM têm-se os diferentes graus de queimadura corporal, um tipo específico de melanoma ou a condição estética antes e após um trauma facial ${ }^{4-6}$.

Além desses exemplos, a estratégia de SRM também pode auxiliar no treinamento de procedimentos, condutas e execução de protocolos padronizados, permitindo avaliar habilidades de comunicação, liderança, tomada de decisão, trabalho em equipe e relacionamento profissional/paciente ${ }^{7}$.

Várias são as dificuldades enfrentadas pelos peritos à avaliação de dano corporal póstraumático, como por exemplo a existência (ou não) da documentação, a antiguidade dos fatos, o nexo causal, a documentação disponível, as simulações, a subjetividade na valoração e a metodologia a ser utilizada. Porém, quando nos deparamos com a valoração do dano estético, temos ainda que comparar um paciente, vítima de trauma com seu estado anterior, ou seja, como ele era antes do evento traumático ${ }^{8}$.

Esta necessidade de conhecimento do estado anterior ao trauma também dificulta a prática do ensino. A busca por alternativas que tornem esse tema mais palpável e acessível para o estudante, assim como alternativas que permitam transpor as peculiaridades éticas nas pesquisas, são de constante interesse para os docentes.

Nesta perspectiva, o presente estudo objetiva relatar uma experiência docente no curso de graduação da Faculdade de Odontologia da Universidade de São Paulo (FO-USP), mostrando o potencial uso da SRM no ensino da valoração de danos pós-traumáticos em Odontologia Legal.

\section{RELATO DE EXPERIÊNCIA}

Inicialmente foi apresentada a proposta de desenvolvimento do conteúdo de valoração do dano corporal pós-traumático por meio da estratégia de SRM para estudantes de graduação matriculados na disciplina de Deontologia Odontológica. A abordagem do conteúdo iniciase por uma aula expositiva sobre a avaliação de dano corporal pós-traumático, quando os alunos recebem informações essenciais sobre o tema, incluindo os parâmetros de danos temporários e permanentes. Destaca-se, dentre os possíveis danos permanentes valoráveis em âmbito civil, o dano estético, tópico onde o método da SRM se apresenta como alternativa. O prejuízo estético deve ser descrito da forma mais detalhada possível e então quantificado por meio de metodologias apropriadas. Então, diferentes métodos foram apresentados aos alunos, dentre eles o método de aferição do impacto e da impressão do prejuízo estético (AIPE). Formaram-se grupos, orientados quanto a simular um determinado grau de prejuízo, com variação de acordo com a nomenclatura do método entre uma lesão de grau "Leve" e uma cicatriz no patamar "Importantíssimo". Posteriormente, a intensidade da lesão devia ser justificada por meio do preenchimento das tabelas do método validado no Brasil para AIPE ${ }^{6}$.

Para simulação das lesões, um estudante de cada grupo teve, de forma voluntária e consentida, a função de modelo, sendo que os demais membros ficaram a cargo da elaboração da maquiagem que simulou os diferentes graus 
de lesões solicitados, sempre com auxílio de material teórico para caracterização das lesões.

Para a maquiagem foram utilizados os seguintes produtos: fio de linha de diferentes espessuras e cores (para simulação de cicatrizes lineares ou complexas), massa de modelar (para confecção de lesões hipersalientes), base líquida e pó (para harmonização com a cor da pele do modelo), bem como materiais de apoio (tesoura, pincel, cola branca lavável, sonda exploradora, pinça, gaze e sabonete líquido para remoção de maquiagem) (figura 1).

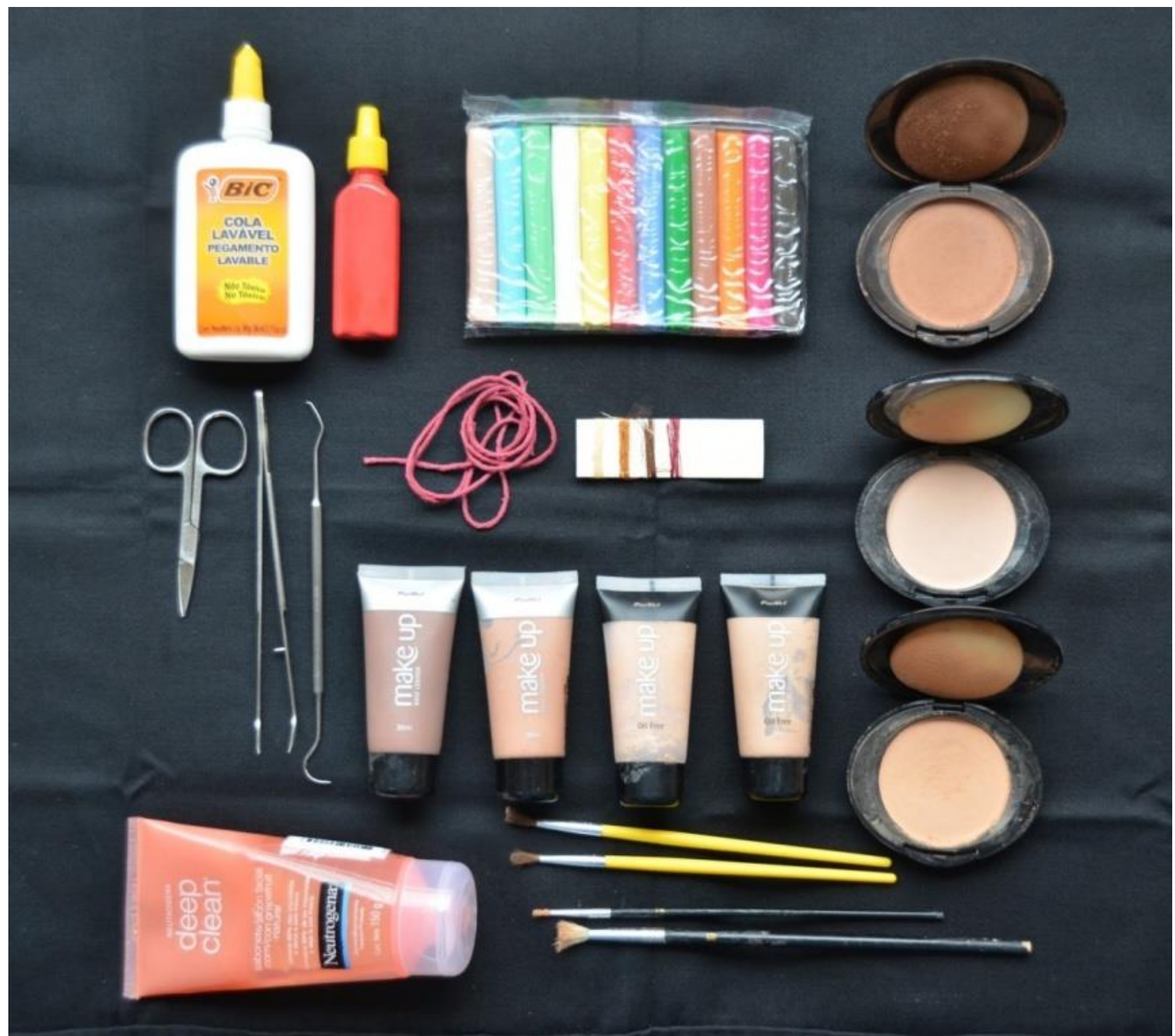

Figura 1 - Materiais utilizados na simulação das lesões

Alguns vídeos demonstram passo a passo como elaborar alguns tipos de lesões corporais mais comuns, conforme a necessidade da simulação ${ }^{9}$. Para o caso ora relatado, utilizou-se a sequência que consta do quadro 1. Para a remoção, foi utilizada gaze e sabonete líquido.

Ao final, cada grupo apresentou seu modelo maquiado com a lesão simulada, explanando como chegou àquela forma, com base nas etapas do método, justificando os 
resultados e as caracterizações realizadas. Tratou-se de uma estratégia-piloto utilizada, quando foi possível perceber o retorno positivo dos estudantes e docentes. Alguns ajustes, tais como a inserção de outros métodos para comparação e outros materiais para simulação podem ser úteis, bem como o feedback através de questionários anônimos.

A estratégia de simulação realística de sequelas por meio de maquiagem, com ou sem posterior ajuste computadorizado, tem sido utilizada no Programa de Pós-Graduação em Ciências Odontológicas, Área de concentração em Odontologia Legal da USP no desenvolvimento de trabalhos sobre valoração do dano corporal pós-traumático envolvendo questões na área de atuação da Odontologia ${ }^{6,10}$. Existem projetos já aprovados e/ou em andamento que, nos casos em que ocorre a participação de alunos, solicita-se consentimento. Nesta linha de pesquisa utilizou-se a maquiagem como método para a avaliação de danos, nos moldes descritos acima para ensino. Esse recurso de imediato viabilizou a padronização de lesões e a comparação dos modelos após o trauma simulado com eles próprios (antes do trauma) como padrãoouro. A elevação gradativa dos elementos constitutivos do dano com a maquiagem já permitiu validar uma escala para quantificação de dano estético em estudo multicêntrico ${ }^{6}$.

Quadro 1 - Sequência de passos para a simulação da lesão

a) Definir o tipo e as características da cicatriz a ser elaborada.

b) Testar a sensibilidade do modelo em relação à cola utilizada, aplicando-a sobre a pele com um pincel.

c) Confirmada a ausência de sensibilidade ao produto, verificar se a cor da base líquida e do pó de maquiagem são compatíveis com a cor da pele do modelo. Caso sejam diferentes, misturar cores até encontrar o tom semelhante.

d) Com auxílio de pinça e sonda, colar a estrutura principal da lesão simulada, que consiste em um pedaço de massa de modelar ou linha grossa de tapeçaria, ambas com a cor compatível, moldando-a com o formato desejado.

e) Colar as porções secundárias que simulam as bordas da cicatriz, utilizando um fio de linha menor e com a cor adequada para simular a cicatriz proveniente da sutura.

f) Aplicar a base líquida de maquiagem com um pincel ao redor da lesão, contornando-a sem cobrir as estruturas principal e a secundária.

g) Aplicar o pó de maquiagem da mesma cor da base líquida sobre toda lesão.

A utilização desse recurso também permite estudar o encadeamento anátomo-clínico das lesões, ou seja, elas podem ser reproduzidas experimentalmente com maquiagem de forma evolutiva na mesma pessoa, abrindo um leque de opções para estabelecer protocolos e critérios de avaliação $^{10}$. A percepção dos diferentes examinadores ao comparar o dano ao estado anterior numa mesma posição (figura 2), passou a ser possível. 

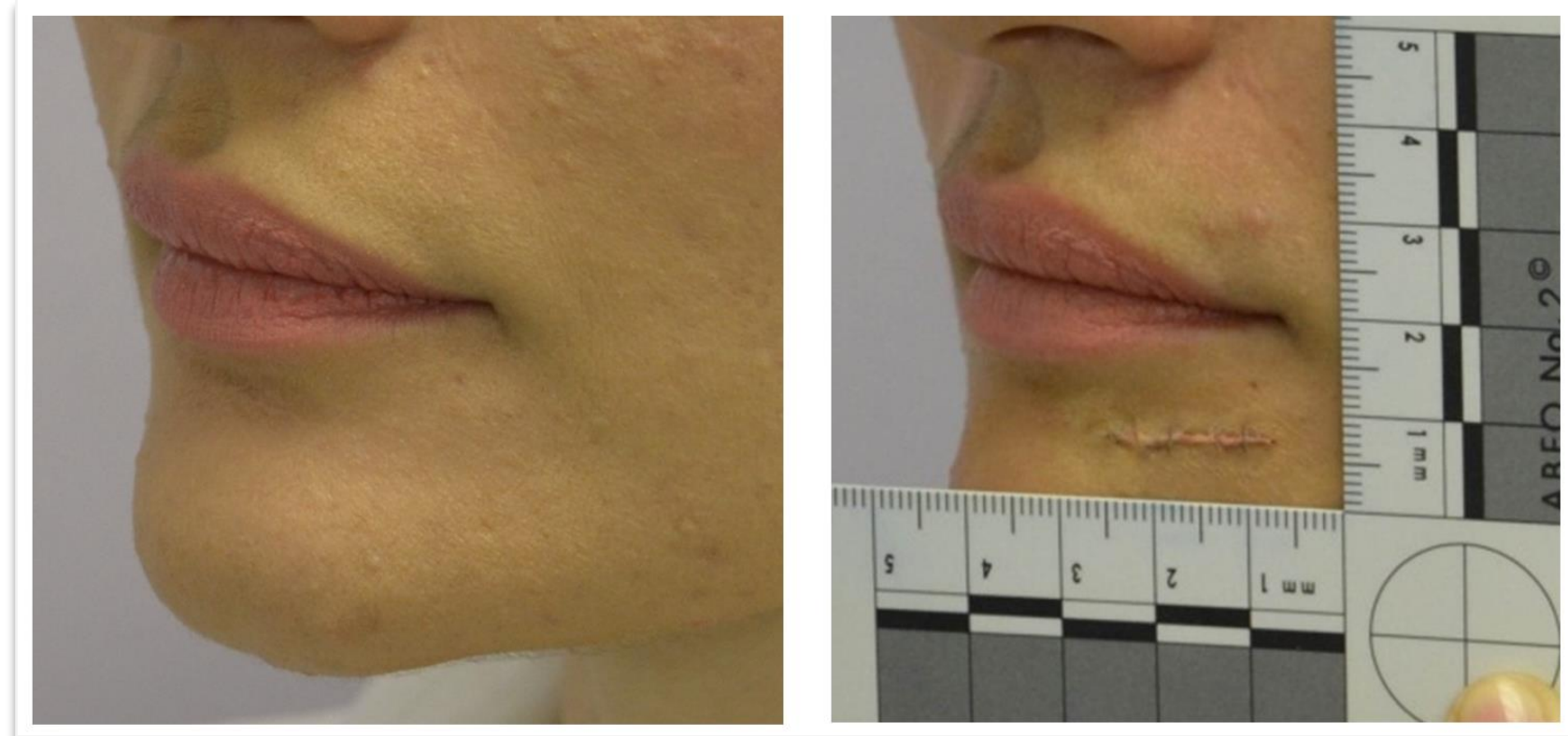

Figura 2 - Modelo fotografado na mesma posição, sem e com maquiagem entre as regiões orbicular dos lábios e mentoniana, simulando cicatriz linear de $2 \mathrm{~cm}$, hipocrômica, hipersaliente e hipersaturada

\section{CONSIDERAÇÕES FINAIS}

Verifica-se, em nível mundial e nos diversos centros de ensino das diferentes áreas da saúde, o uso da maquiagem como recurso para percepção de lesões, como no ensino da dermatologia, em que diferentes lesões simuladas são aplicadas ou elaboradas sobre a pele, incluindo pequenas próteses ${ }^{11}$, bem como no ensino da enfermagem, na abordagem de casos no ambulatório de emergências, conforme o tipo de lesão 9 .

Metodologias ativas estão cada vez mais presentes em salas de aula devido ao fato de estimularem o aprendizado crítico-reflexivo e a interação entre discentes e docentes. Nesse contexto, o aprendizado baseado em problemas incentiva o pensar e o fazer, apresentando melhores resultados que os métodos tradicionais $^{12}$.

$\mathrm{O}$ uso da maquiagem enriquece o ensino, aproximando o estudante da prática pericial relacionada à valoração do dano estético, um tema complexo que se torna palpável e de mais fácil assimilação. $\mathrm{Na}$ experiência relatada, mais do que se deparar com o caso pronto, o estudante teve que, a partir de parâmetros propostos, construir seu modelo/paciente padronizado.

Esse processo permitiu, de maneira lúdica, porém fundamentada em princípios teóricos consolidados, o aprofundamento do aprendizado, uma vez que foi necessária interação entre os membros de cada grupo, na revisão de conceitos e parâmetros para chegarem ao correto grau de lesão a ser simulada. Existe uma crescente preocupação, não somente com as metodologias que serão utilizadas em sala de aula, mas também com que as IES abordem assuntos relacionados à Odontologia Legal e a ofertem como disciplina curricular. É de grande importância que os alunos de graduação tenham, além do conhecimento técnico científico, ciência dos preceitos éticos e legais para que possam aplica-los no decorrer da sua vida profissional ${ }^{13}$.

No caso da valoração de danos permanentes, mais especificamente nas situações que envolvam o dano estético, é fundamental 
considerar as características da pessoa afetada e os elementos constitutivos do dano como localização, morfologia, dimensões, orientação e coloração. Também se deve levar em consideração a idade, sexo, profissão e sensibilidade do lesionado ao dano estético sofrido. Finalmente, há que valorar a possibilidade de reparo do dano estético e os possíveis riscos e resultados desse tratamento ${ }^{8}$.

Em relação ao nível de impacto do prejuízo estético, essa técnica permite escalonar de forma mais refinada as categorizações de dano, tornando quantificável o que foi, até então, subjetivo $^{6,8}$.

Manequins com cabeça e pescoço de borracha são menos fidedignos que a simulação em modelos na prática de avaliação pericial. A simulação em modelos facilita a visualização, assimilação e fixação das nomenclaturas conforme o tipo de cicatriz elaborada.

Dessa forma, ressalta-se a importância da incorporação de metodologias de ensino mais dinâmicas, lançando mão de recursos educacionais interativos e motivando, assim, o aprendizado moral, ético e técnico-científico ${ }^{14}$.

$\mathrm{O}$ recurso da maquiagem como ferramenta educacional proporcionou o efetivo envolvimento dos alunos na atividade de avaliação de dano corporal, num processo lúdico e reflexivo para a construção do modelo/paciente padronizado. A simulação realística, na modalidade maquiagem, mostrou-se estratégia promissora para o campo das pesquisas forenses, com alto potencial de uso no ensino da Odontologia Legal.

\section{ABSTRACT \\ Moulage as teaching and research strategy in Forensic Dentistry: experience report \\ In many situations, the teaching of the posttraumatic dental damages valuation is carried out in a theoretical and illustrative way using projected images, distancing the student from the}

practical experience of this course. For this reason, simulated lesions using moulage can help to teach this topic, as well as in research in the field, considering, for example, the need to compare the trauma victim with his previous condition. The objective of this article is to report a teaching experience with the undergraduate Dental degree program, Universidade de São Paulo, highlighting the potentiality of realistic simulation with moulage in teaching valuation of posttraumatic injuries and encouraging its possible application in Forensic Dentistry research. This is an innovative methodology, showing a promising perspective in the field of Forensic Dentistry.

Descriptors: Damage Assessment. Damage Assessment Methodologies. Forensic Dentistry. Esthetics. Liability, Legal.

\section{REFERÊNCIAS}

1. Haddad AE, Laganá DC, Assis EQ, Morita MC, Toledo AO, Rode SM, et al. A aderência dos cursos de graduação em Odontologia às Diretrizes Curriculares Nacionais. In: Ministério da educação. A aderência dos cursos de graduação em Enfermagem, Medicina e Odontologia às Diretrizes Curriculares Nacionais. Brasília; 2006. 119-152. [Acesso em 27 set. 2016]. Disponível em: http://abeno.org.br/ arquivos/downloads/download_201112151 03241.pdf.

2. Beaini TL, Zanin AA, Miranda GE, Flores M, Curi JP, Melani RFH. Dinâmica de perícia em local de crime na disciplina de Odontologia Forense da FO-USP. Rev Grad USP. 2016; 1(1):77-82.

3. Oliveira RN, Mazzilli, LEN. Fomentando o olhar crítico da realidade odontológica. In: Anais do PBL2016 International Conference. São Paulo; 2016. p. 41.

4. Pywell MJ, Evgeniou E, Highway K, Pitt E, Estela CM. High fidelity, low cost moulage as a valid simulation tool to 
improve burns education. Burns. 2016 Jun;42(4):844-52.

5. Wang S, Seelaus R, Rea CA, Hernandez C. Use of melanoma simulation model in a dermatology objective structured clinical examination station. Med Teach. 2015 Feb;37(2):202-3.

6. Fernandes MM, Cobo Plana JA, Bouchardet FHC, Michel-Crosato E, Oliveira RN. Validação de instrumento para análise do dano estético no Brasil. Saúde Debate. 2016; 40(108):118-30.

7. Brandão CFS, Collares CF, Marin HF. A simulação realística como ferramenta educacional para estudantes de Medicina. Sci Med. 2014; 24(2):187-92.

8. Plana JAC. La valoración del daño a las personas por accidentes de tráfico. Barcelona: Bosch; 2010, p 635-706.

9. Smith-Stoner M. Using moulage to enhance educational instruction. Nurse Educ. 2011; 36(1):21-4.

10. Fernandes MM, Baldasso RP, Sakaguti N, Bouchardet FCH, Plana JAC, Oliveira RN. Como justificar a ausência de dano estético? Relato de perícia civil odontológica. RBOL. 2017; 4(1):114-21.
11. Garg A, Haley HL, Hatem D. Modern moulage: evaluating the use of 3dimensional prosthetic mimics in a dermatology teaching program for secondyear medical students. Arch Dermatol. 2010; 146(2):143-6.

12. Queiroz JRC; Oliveira MFJ; Souza DMC; Araújo ALLS; Fuscella MAP. Aprendizagem por projeto e inovação tecnológica: união por competências. Rev ABENO. 2016; 16(2):2-6.

13. Brites AN, Pithan SA, Nunes MF, Brites IF. Odontologia Legal no ensino superior do Estado do Rio Grande do Sul. Rev ABENO. 2016; 16(3):36-45.

14. Lino-Júnior HL, Gabriel M, Daruge-Junior E, Silva RHA. Ensino de Odontologia Legal no Brasil: um convite à reflexão. Rev ABENO. 2015; 15(2):38-46.

\section{Correspondência para:}

Mário Marques Fernandes

e-mail: mfmario@usp.br

Departamento de Odontologia Social, Universidade de São Paulo

Prof. Lineu Prestes 2227 - Cidade Universitária 05508-000 - São Paulo, SP 